\title{
Early intervention at home in infants with congenital brain lesion with CareToy revised: a RCT protocol
}

\author{
Giuseppina Sgandurra ${ }^{1,2^{*}}$, Elena Beani ${ }^{1}$, Matteo Giampietri ${ }^{3}$, Riccardo Rizzi ${ }^{4}$, Giovanni Cioni $^{1,2}$ \\ and the CareToy-R Consortium
}

\begin{abstract}
Background: Congenital brain lesions expose infants to be at high-risk for being affected by neurodevelopmental disorders such as cerebral palsy (CP). Early interventions programs can significantly impact and improve their neurodevelopment. Recently, in the framework of the European CareToy (CT) Project (www.caretoy.eu), a new medical device has been created to deliver an early, intensive, customized, intervention program, carried out at home by parents but remotely managed by expert and trained clinicians. Reviewing results of previous studies on preterm infants without congenital brain lesion, the CT platform has been revised and a new system created (CT-R).

This study describes the protocol of a randomised controlled trial (RCT) aimed to evaluate, in a sample of infants at high-risk for CP, the efficacy of CT-R intervention compared to the Infant Massage (IM) intervention.

Methods/design: This RCT will be multi-centre, paired and evaluator-blinded. Eligible subjects will be preterm or fullterm infants with brain lesions, in first year of age with predefined specific gross motor abilities. Recruited infants will be randomized into CT-R and IM groups at baseline (T0). Based on allocation, infants will perform an 8-week programme of personalized CareToy activities or Infant Massage. The primary outcome measure will be the Infant Motor Profile. On the basis of power calculation, it will require a sample size of 42 infants. Moreover, Peabody Developmental Motor ScalesSecond Edition, Teller Acuity Cards, standardized video-recordings of parent-infant interaction and wearable sensors (Actigraphs) will be included as secondary outcome measures. Finally, parents will fill out questionnaires (Bayley SocialEmotional, Parents Stress Index). All outcome measures will be carried out at the beginning (T0) and at end of 8-weeks intervention period, primary endpoint (T1). Primary outcome and some secondary outcomes will be carried out also after 2 months from $\mathrm{T} 1$ and at 18 months of age (T2 and T3, respectively). The Bayley Cognitive subscale will be used as additional assessment at T3.
\end{abstract}

Discussion: This study protocol paper is the first study aimed to test CT-R system in infants at high-risk for CP. This paper will present the scientific background and trial methodology.

Trial registration: NCT03211533 and NCT03234959 (www.clinicaltrials.gov).

Keywords: Early intervention, High-risk infants, Randomized clinical trial, Tele-rehabilitation, Information and communication technology, Neurodevelopmental bioengineering, Cerebral palsy, Infant massage

\footnotetext{
* Correspondence: g.sgandurra@fsm.unipi.it

${ }^{1}$ Department of Developmental Neuroscience, IRCCS Fondazione Stella Maris,

Viale del Tirreno 331, Calambrone, 56128 Pisa, Italy

${ }^{2}$ Department of Clinical and Experimental Medicine, University of Pisa, Via

Roma, 56125 Pisa, Italy

Full list of author information is available at the end of the article
}

(c) The Author(s). 2018 Open Access This article is distributed under the terms of the Creative Commons Attribution 4.0 International License (http://creativecommons.org/licenses/by/4.0/), which permits unrestricted use, distribution, and reproduction in any medium, provided you give appropriate credit to the original author(s) and the source, provide a link to the Creative Commons license, and indicate if changes were made. The Creative Commons Public Domain Dedication waiver (http://creativecommons.org/publicdomain/zero/1.0/) applies to the data made available in this article, unless otherwise stated. 


\section{Background}

Cerebral Palsy (CP), a clinical outcome linked to pre- or perinatal brain injury, represents the main chronic condition of disability in childhood [1, 2]. Disorders and disabilities associated with CP determine a relevant social, financial and emotional influence, on $\mathrm{CP}$ subjects, on their relatives, and also on health services, since these patients require continuous care, treatment and social support throughout their entire lives [3, 4]. The combined use of assessment tools such as the General Movement Assessment (GMA) according to Prechtl (GMs) and brain Magnetic Resonance Imaging (MRI) have shown high sensitivity and specificity in CP identification in infants since their first months of life [5]. In particular, a recent literature review has shown that brain MRI associated with GMA or neurological examination (Hammersmith Infant Neonatal Examination, HINE) performed at around full-term age determines the greatest predictive power of CP in high-risk newborns [6]. Early diagnosis has considerable importance because it allows for an early medical response and intervention which, as indicated by literature, can improve developmental outcome of high-risk children [7]. Moreover, early intervention (EI) is crucial because it targets brain plasticity, which for many functions has a maximum expressivity in an early limited time window or "critical period" $[8,9]$.

Several EI programs, based on Environmental Enrichment (EE) and Goal Directed approaches, have been used in clinical settings with positive results on neurodevelopment, but these findings are not conclusive due to high heterogeneity of clinical studies and applied intervention models [10-13].

In general, EIs should include certain essential characteristics to be effective and more specifically they should be early, intensive, personalized, multi-axial, family-centred and affordable for families and health services. In this context, biotechnologies and tele-rehabilitation appear to be promising approaches that can help achieve these standards [14].

Recently, in a European project (www.caretoy.eu, Trial Registration: NCT01990183), the CareToy (CT) system has been created. It is a technological and modular system able to provide, by means of tele-rehabilitation, a home and personalized intervention for very young infants (for further details see $[15,16]$ ). CT allows subjects to carry out an early, intensive, individual EI carried out at home by parents consisting of highly customized exercises (rehabilitative packages called scenarios) remotely monitored by a clinical staff [15-18].

$\mathrm{CT}$ has been recently validated as a EI tool through a RCT study (CareToy training vs Standard Care), preceded by a pilot study, which involved a total of 61 in preterm infants born from 28 to 32 weeks of gestational age, without brain lesions and therefore considered at low risk for neurodevelopmental disorders. Children recruited in the pilot and RCT studies were divided into CT and Standard Care groups. Children allocated to the CareToy group followed a 4 weeks training with CT system, at the same time children allocated to the control group performed only Standard Care. All children were assessed with specific and standardized scales and questionnaires, immediately before and immediately after treatment period. Results of the study showed that CareToy training has a positive effect on promoting short-term visual and motor infant's development $[19,20]$.

Based on these findings, the purpose of this study is to compare, through a Randomized Controlled Trial (RCT), the effects of two types of EI (CareToy training vs Infant Massage) on neurodevelopment of a group of at least 42 children at high risk for CP. The general hypothesis is that the CareToy system, with some adaptations, could be a useful EI tool also in brain-lesioned children at high risk for $\mathrm{CP}$, effectively promoting motor, cognitive and perceptual development. In order to provide an EI to all children participating in the study, it was decided to offer Infant Massage (IM) as a valid alternative to CareToy. The choice of proposing IM was favoured by its ever-increasing application in Neonatal Intensive Care Units (NICU) [21]. The general hypothesis of IM intervention is that tactile stimulation (parent-infant) is able to promote neurodevelopment, emotional regulation of behavioural states and parent-infant relationship [22]. The rationale underlining these mechanisms seems attributable to increased metabolic efficiency and reduction of stress hormone synthesis. A recent systematic review [22] highlighted the effectiveness of IM on promoting neurodevelopment of preterm babies [23]; moreover, although studies on IM in infants with early brain damage are still limited, it seems to have positive effects on muscle tone and general motor development [24].

Based on this state of the art, the main purpose of this study is to evaluate the effects of CareToy EI (with a revised version of CareToy designed for this purpose), compared to those of Infant Massage, on neurodevelopment of infants at high risk for CP.

\section{Methods/design}

This paper presents the protocol of an RCT which compares the effects of CareToy training to those of Infant Massage on neurodevelopment of infants at high risk for CP. Details of the two treatment protocols are described below.

\section{Ethical considerations}

Tuscan Region Paediatric Ethics Committee (Italy) approved this study (no. 84/2017). Before the signing of informed consent, a dedicated personnel will verbally inform all parents of eligible infants about the trial, 
giving them also written informative material. Two informed consent forms during two different phases of the study will be provided. The first form allows for an observational phase related to a standardized GMA from the writhing period up to the fidgety one. Then, a second consensus form related to the intervention phase (CareToy or IM) will be given only to parents of infants with fidgety absent. There is a dual purpose for offering two treatments: to compare the two EIs and, for ethical reasons, to allow all eligible infants to carry out an EI.

\section{Primary objective}

The main aim of the present trial is to explore the effects of CT training on neurodevelopment of infants at high risk of $\mathrm{CP}$ and then compare these effects to those of IM.

Three hypotheses have been specified:

1. CareToy Revised (CT-R) is a useful rehabilitative medical device for young infants $(<1$ year) at high risk for $\mathrm{CP}$

2. Infants receiving CT-R training will develop motor, perceptual and cognitive abilities faster than infants receiving IM

3. Improvement in visual and motor abilities will be faster during CT-R training than during IM.

The secondary aim is to investigate the different impact of CT-R on neuromotor development, both quantitatively (postural, motor and manipulation competences) and qualitatively (motor repertoire and adaptive abilities). Moreover, another purpose is to measure the efficacy of CT-R training on visual and cognitive development, parent-infant interaction and sleep-waking pattern.

The two phases of this project will be preceded by a small pilot study in which feasibility of EI effects of CareToy Revised system (CT-R training) compared with Infant Massage (IM) will be evaluated.

\section{Study design}

To make a comparison between the effects of CT training and IM in brain-damaged infants, a multicentre, evaluator-blinded, paired RCT will be carried out.

The involved clinical centres are the Department of Developmental Neuroscience, IRCCS Fondazione Stella Maris in Pisa; the Neonatal Intensive Care Unit of University Hospital "Santa Chiara" in Pisa, the Neonatal Intensive Care Unit, Department of Perinatal Medicine of University Children's Hospital "A. Meyer" and the Division of Neonatology, Careggi University Hospital, in Florence.

There will be two investigative arms: CT-R and IM training. Both training programs will last 8-week.
All the infants will be clinically assessed at T0 (baseline, in the week preceding CT-R training/IM) and at T1 (i.e. in the week after the end of the 8-week programme of CT-R training/IM). T1 will be the primary endpoint. Then, all infants will be followed at T2 (i.e. 8 weeks after the end of intervention period) and at T3 (i.e. at 18 months of age). Figure 1 shows the detailed timeline.

This study will be structured in two parts: an observational phase and an intervention one.

The observational phase will be aimed to early detect the brain-lesioned infants at high risk for $\mathrm{CP}$ through standardization of traditional clinical procedures, based on the most updated existing criteria. The following intervention phase will be aimed at verifying and comparing, with a RCT, the efficacy of two EI models (CareToy-Revised and Infant Massage) in promoting neurodevelopment of high-risk infants.

\section{Observational phase}

The observational phase will consist of a standardized follow-up for monitoring development of infants through ultrasound evidence of brain injury verified at the two Neonatology Units involved in this project. Monitoring will also include neurological assessment (HINE) and recording of spontaneous activity according to Prechtl (GMA) [25, 26].

These examinations will be performed at around term period and also 2-3 months after post-term age, in the Neonatology Unit designated for regular clinical follow-up examinations.

Moreover, as clinical recommended for high-risk infants, a brain MRI during spontaneous sleep will be performed within 6 weeks post-term. MRI protocol foreseen by this project includes structural images, already present in clinical protocols adopted by the involved clinics and necessary for definitive inclusion in the second intervention phase, and a brief period of functional MRI acquisition [27-29]. It will include two further series of images (GRE-EPI, TR $/ \mathrm{TE}=3000 / 50, \mathrm{FA}=90^{\circ}, \mathrm{FOV}=240 \times$ $240 \mathrm{~mm}$, matrix $=96 \times 96$, thickness $=3 \mathrm{~mm}$ ), for a total duration of 4 minutes, according to a block diagram, with an alternation of visual stimulus presentation suitable for assessing visual system integrity that is used in movement perception (MT area of the parietal cortex). More specifically, the passive stimulus consists of image presentations, through appropriate non-magnetic glasses, for MRI that simulates coherent or incoherent movement.

\section{Intervention phase}

In the interventional phase, infants will proceed with an early intervention, which could be CareToy-R or Infant Massage, according to allocation group. Each intervention will last 8 weeks, 5 days a week; during this experimental phase all infants will continue to receive 


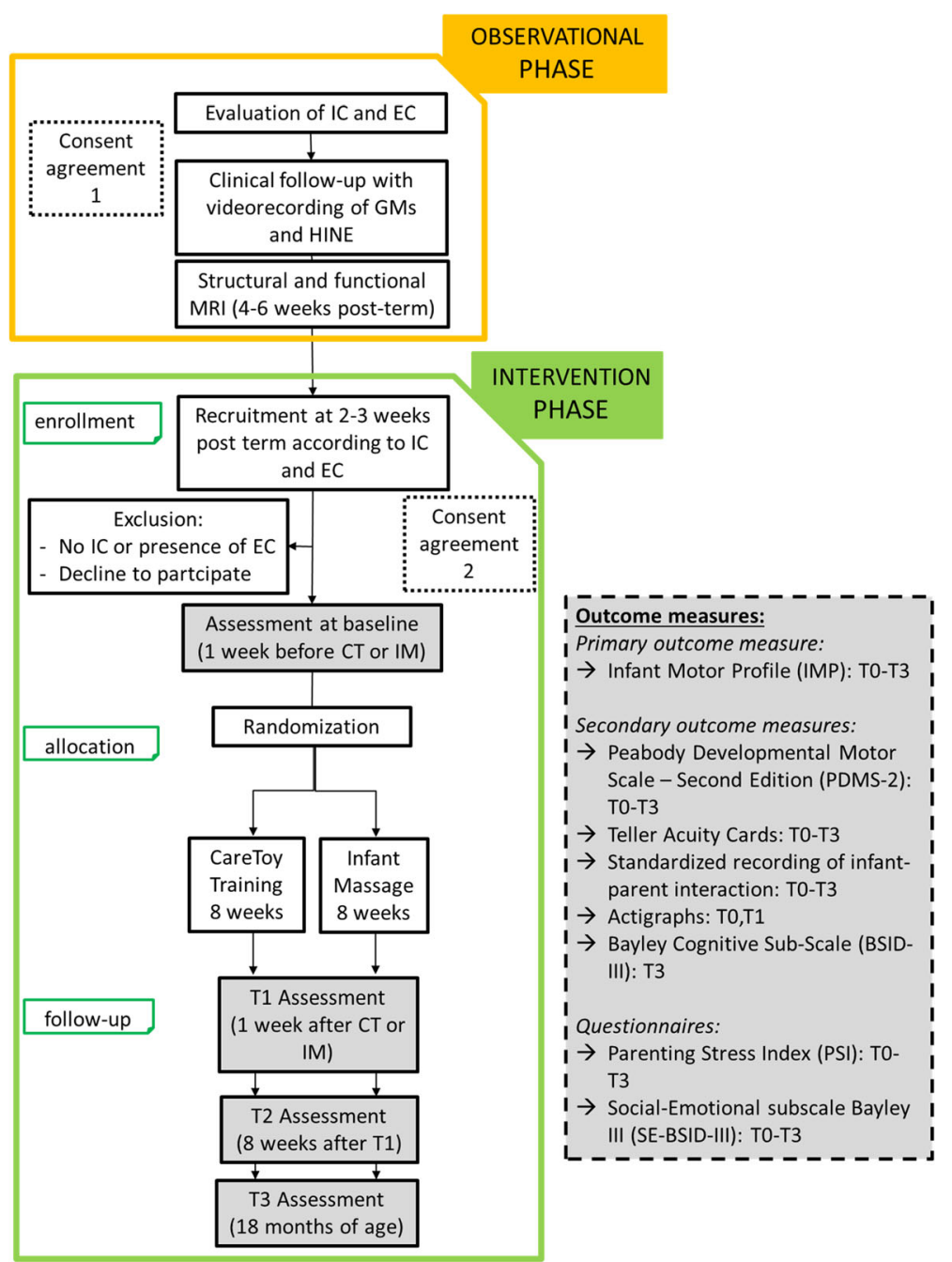

Fig. 1 Study design

standard care foreseen by National Health System. All other specific interventions (such as physical therapy or other treatments) will be recorded in a specific diary. Therapy protocols are described below.

\section{Study sample and recruitment}

Participants will be recruited at Tuscany Neonatology Units in Pisa (Santa Chiara University Hospital) and in Florence (Meyer and Careggi Hospitals). The neonatological staff will assess eligibility of infants on the basis of inclusion and exclusion criteria prior to hospital discharge and staff will inform parents about study. If a family expresses interested in participating in the study, they will receive an introductory letter and an informative flyer. Recruitment must take place within the first year of age of the infant and, because of the characteristics of the interventional setting and exercises, before the complete acquisition of the trunk control in sitting position. If parents are willing to participate, they will be asked to sign a first agreement for admission to the observation phase and another one to partake in the intervention phase. As indicated above, Tuscan Region Paediatric Ethics Committee approved the clinical trial. Moreover, CT-R, as the previous CT, has been classified as a medical device without an EC mark, so an approval of a new clinical trial was requested and obtained by the Italian Ministry of Health.

Inclusion criteria will be the following:

- Abnormal or specific neurological signs at neurological examination of GMA or HINE, associated with one of the following conditions

- Persistent brain ultrasound periventricular hyper-sonority

- Evidence of cerebral haemorrhage

- Evidence of Periventricular Leukomalacia (PVL)

- Cerebral stroke 
- Moderate or severe asphyxia

\section{Exclusion Criteria:}

- Polymalformative syndrome or cerebral malformations

- Severe retinopathy (III-IV degree)

- Severe sensory disturbances (deafness or blindness)

Moreover, to be enrolled in the intervention phase, additional inclusion criteria will be:

- Persistence of abnormal Spontaneous GMs evaluation according to Prechtl at 2-3 months postterm (absent or abnormal FMs)

- Persistence of abnormal/specific signs according to HINE

Finally, the inclusion criterion for the start of intervention phase (CareToy- $\mathrm{R}$ or Infant Massage) is based on motor requirements defined on the basis of Ages \& Stages Questionnaire scores. In general, skills required to start EI range from initial head control to complete trunk control in sitting position. In other words, infants who have no initial head control or have acquired the sitting position cannot be admitted to the intervention phase. In the first case, it is necessary to wait until the infant can control the head within the first year of age, in the second case they will not allocated to the intervention phase.

To monitor motor progress, families will receive the section on "gross motor skills evaluation" of Ages \& Stages Questionnaire (ASQ-3; gross motor [30]); as soon as the child reaches some pre-established motor skills (see below). Treatment can then start according to assignment group.

- score $\geq 10$ from 3 to 4 months

- score $\geq 5<50$ from 5 to 6 months

- score $\geq 10<30$ from 7 to 8 months

The exclusion criterion is represented by a worsening of general clinical situation for intercurrent medical conditions or an onset of epileptic seizures.

\section{Sample size}

Based on the chosen primary outcome measure, Infant Motor Profile (IMP [31],), and on IMP results of a pilot study and of previously conducted RCT in preterm infants without congenital brain lesions $[15,19,20]$ and considering a $80 \%$ power and a significant level of 0.05 and a effect size of at least 0.6 , a sample of 38 children is required. However, considering also a $10 \%$ of drop-out rate, a minimum of 42 infants will be required. In order to facilitate parental work and participation in the project, eligible twins will be allocated into the same group.

\section{Randomisation}

Randomization will be done after enrolment: participants will be randomly assigned to CT-R training or IM group by the use of an automatic generation of 1:1 sets. A third party blind to clinical aspects of trial will manage these sets, sealing them in numbered envelopes.

\section{Blinding}

Parents, therapists and clinical staff will be aware about the allocation group of infants. Assessors who will evaluate infants with outcome measures (IMP; Peabody Developmental Motor Scales - Second Edition (PDMS-2); Bayley Scales of Infant Development III Edition, BSIDIII) will use the video-recording of the assessment session so they will be absolutely blind.

\section{Therapy protocols \\ CareToy- $R$ training}

As in a previous study [20], the clinical staff, mainly composed by child neurologists and paediatric physical therapist will organize customized goal-directed rehabilitative activities (i.e. CareToy scenarios) to be done in three different possible position which could be sitting, supine or prone, mainly focused on: postural abilities (e.g. postural control, rolling...), manipulation capabilities (e.g. reaching, grasping, manipulation...) and visual functions (e.g. visual attention and orientation...).

The new CT system, i.e. the revised version (CT-R), has been planned and designed in order to adapt the CareToy system to this new population by integrating a support for posture maintenance. Based on postural needs of brain-lesioned infants, a modular system called Siedo \& Gioco (Fumagalli, Italy) has been incorporated. Siedo \& Gioco system is a soft multifunctional system which allows the infant or a small child to be placed in many different confortable and safe positions (e.g. supine, prone, sitting or on one side). The Siedo \& Gioco system is composed a set of soft and coloured modules with different shapes which can be attached to a mat by Velcro straps placed on modules and on mat. Technical specification for optimize suitability of Siedo \& Gioco to CT system have been provided and an ad-hoc set of modules has been created in order to offer postural and perceptive stimuli. Thanks to the flexibility of this integrated system, the modularity of CT platform in this revised version (CT-R) has been maintained.

According to the initially established activities and modules (the activities and consequently the modules will be periodically updated), the CT-R system will be customized and delivered to the family's home. CT-R 
training will be structured in 8 weeks, in which there will be about 30-45 min of daily planned activities, consisting of different scenarios lasting 2-10 min each.

Parents will be instructed to carry out the daily training during the wake and active periods of their infants. It will be remotely supervised by the clinical staff, who will have the possibility to remotely manage the CT scenarios based on each infant rehabilitation requirement and improvement during the 8-weeks training. Each scenario will be scored at the end by the parents on the basis of a questionnaire recording the infant's acceptance and compliance.

Rehabilitation staff will train parents to use the system and to interact with their infant during the first 5 days of training and once a week in the following weeks.

\section{Infant massage}

Infant massage consists generally of a systematic touching by hands on different parts of the body and is characterized by slowness and gentleness [22]. IM is often associated to other forms of "contact" with the infant, e.g. kinaesthetic (arm and leg passive extension/flexion), auditory, verbal or visual stimuli.

Families assigned to IM group will perform a training course of 4-5 sessions lasting about one hour each. The massage will be done on different body areas and illustrated material on how to perform massage sessions at home will be provided to families. Parents will be required to perform IM 5 days a week, depending on the child's willingness, for 8 weeks, and to record massage frequency in a dedicated diary.

\section{Outcome measures}

As previously reported, infants will be assessed according to the timeline (Fig. 1). The primary outcome measure, assessing the motor development, will be the IMP. The selected secondary outcomes will be the: PDMS-2, BSID-III Cognitive subscale, standardized video-recordings of parent-infant interaction, Teller Acuity $\mathrm{Cards}^{\circ}$ and Actigraphic analysis (Motionlogger Microwatch). Due to multiple outcomes and variable willingness of infants, each evaluation time would be completed, if necessary, in two successive days to guarantee greater compliance.

\section{Primary outcome measure Infant motor profile (IMP)}

IMP is a video-recorded motor evaluation of the infant placed in different positions (supine, prone, sitting, standing while grasping and manipulating objects). It is composed at first by observation of spontaneous activity and then by stimulation of motor abilities and manipulation of objects in different position. It consists of 80 items, subdivided in five motor domains, which allow for a calculation of a total IMP score. IMP does not only evaluate motor performance in quantitative terms, but also provides qualitative assessments, such as movement variability and fluency and adaptability of motor strategies.

It is suitable for infant born at term and preterm from 3 months of age to until the child has acquired good autonomous skills (which is approximately 18 months).

Previous studies with CT have had the IMP as the primary outcome measure. It will be carried out at all assessments as shown in Fig. 1.

\section{Secondary outcome measures}

According to secondary goals, the following measures have been selected.

\section{Motor assessment \\ Peabody developmental motor scales - second edition (PDMS-2)}

It evaluates fine and gross motor movements from birth to 5 years. The scale is made up of 6 subtests (reflex, sitting, walking, manipulation of objects, grasping and visual-motor integration), whose results are combined into 3 global motor performance quotients: Gross Motor, Fine Motor and Global Motor [32]. It will be performed at all time-points (T0, T1, T2 and T3).

\section{Cognitive assessment BSID-III-cognitive subscale}

It is a standardized scale for the cognitive development assessment in 1-42 months old children [33]. It will be performed at $\mathrm{T} 0$ and $\mathrm{T} 3$.

\section{Visual function assessment Teller acuity cards $^{\circledast}$}

It is a test widely used in young infants and non-collaborative subjects to estimate visual acuity. The trained assessor proposes a series of black and white stripes cards with different widths on one side and neutral stimulus (grey background) on the other side to the infants, judging his/her visual attention. It evaluates the skill to look at a visual target. The estimated visual acuity is the thinnest width of stripes that the child is able to fix and prefer to the neutral grey area. The test is based on the "preferential looking" concept; response indicators are on a spontaneous behaviour basis, i.e. head positioning and eye directing to stimulus. The test is highly reliable, versatile, and it can be executed in a few minutes [34, 35]. It has been used in scientific studies for evaluating the results of training on visual development [36]. It will be performed at T0, T1, T2 and T3. A further complementary visual assessment will be carried out by means 
of an eye-tracker (CareToy C) [37] that allows a measurement of fixation and pursuit.

\section{Parent-infant relationship assessment Standardized video-recordings of parent-infant interaction} A video of a free play interaction of about 3-5 min between parent and child will be carried out. Videos will be classified by expert certified raters according to Child Adult Relationship Experimental Index (CARE - Index) [38] and/or Emotional Availability (EA) Scales [39], blinded to allocation group. They will be carried out at T0, T1, T2 and T3.

\section{Study of organization and maturity of sleeping Movement recording with Actigraphs}

Actigraphs is the simplest and least invasive measurement tool to evaluate sleep which allows for protracted monitoring (from days to months) [40]. It uses accelerometric sensors similar to a wristwatch and it is generally worn on the non-dominant side (hand or wrist). Motionlogger Microwatch will be used and combined with Sadeh algorithm for sleep evaluation. Clinical data such as total sleep time (TST), waking after sleep onset (WASO), and sleep efficiency (SE) will be calculated during the training. It will be worn on an infant's ankle for 1 week at $\mathrm{T} 0$ and $\mathrm{T} 1$.

\section{Questionnaires}

\section{BSID-III social-emotional scale}

This is a subscale of the Bayley III. It is a screening tool for early identification of social-emotional deficits in subjects from birth to 42 months. It is a questionnaire to be completed by parents and it allows an evaluation of child emotional-functionality, communicative needs, interactive relationship and use of interactive emotions to social problem-solving [41]. This scale will be done by the parents at T0, T1, T2 and T3.

\section{Parenting stress index (PSI)}

This questionnaire, containing 36 items, is divided into 3 subscales and requires about 5-10 min to fill out. It detects defined features of parents, of their children and of their environment that are frequently related to parenting stress. It is a tool, widely used for early detection of parent-child dysfunction in their relationships [42]. It will be filled in by the parents at T0, T1, T2 and T3.

\section{Analyses}

Statistical Package for Social Sciences (SPSS) will be adopted for carrying out the statistical analyses. Descriptive statistics will be performed to create the record of the data for each group. Potential baseline differences and between group differences will be explored computing the $p$ values. When necessary, Bonferroni correction will be applied. Firstly, delta changes immediately after treatment (T1, primary endpoint) vs baseline will be calculated to assess the short-term effects of CT-R training versus IM in primary and secondary outcome measures, taking account as covariates base level of motor development, type and grading of brain lesion, family compliance and dose of intervention. After, multivariate statistics will be performed.

\section{Discussion}

This paper shows rationale and protocol for an RCT aimed at evaluating the efficacy of a new EI tool, called CT-R, respect to IM in brain-damaged infants.

It is built on several studies carried out over the last years $[15,17-20,43]$ in infants without brain lesions. According to our experience, there are some limitations in performing high-quality home-based EI that could be different from family to family because home environments are various and difficult to control. On the other hand, the advantages for families are that they do not have to go to a clinic for every intervention session and they can personally learn how to stimulate their infant in their own home. In our proposal, the abovementioned disadvantages can be overcome thanks to the option of remotely managing home-training from the clinic, in this way parent behaviour can be controlled and EI will have greater access.

CT-R system is a completely new technological tool for delivering at home personalized EI in infants. The precise study protocol, that follows the CONSORT guideline $[44,45]$ and the comparison with another EI, will permit to scientifically assess the effects of CT-R intervention on neurodevelopment in a sample of brain-lesioned infants.

\section{Abbreviations}

ASQ-3: Ages \& Stages Questionnaire - 3; BSID-III: Bayley Scales of Infant Development - III; CARE Index: Child - Adult Relationship Experimental Index: CP: Cerebral Palsy; CT: CareToy; CT-R: CareToy Revised; EE: Environmental Enrichment; El: Early Intervention; FMs: Fidgety Movements; GMA: General Movement Assessment; GMs: General Movements; HINE: Hammersmith Infant Neonatal Examination; IM: Infant Massage; IMP: Infant Motor Profile;

MRI: Magnetic Resonance Imaging; NICU: Neonatal Intensive Care Units; PDMS2: Peabody Developmental Motor Scales - Second Edition; PSI: Parenting Stress Index; RCT: Randomised Controlled Trial

\section{Acknowledgements}

The CareToy-Revised Project Consortium, in addition to the authors of this study is composed by:

Francesca Cecchi, The BioRobotics Institute, Scuola Superiore Sant'Anna, Pisa, Italy.

Maria Luce Cioni, AOU "A. Meyer" Firenze, Italy.

Carlo Dani, Division of Neonatology, Careggi University Hospital of Florence, Florence, Italy.

Paolo Dario, The BioRobotics Institute, Scuola Superiore Sant'Anna, Pisa, Italy. Marco Di Galante, IRCCS Fondazione Stella Maris, Calambrone, Pisa, Italy. Ugo Faraguna, IRCCS Fondazione Stella Maris, Calambrone, Pisa, and

Department of Translational Research and of New Surgical and Medical Technologies, University of Pisa, Italy.

Patrizio Fiorini, AOU "A. Meyer" Firenze, Italy. 
Paolo Ghirri, AOU "Santa Chiara", Pisa, and Department of Clinical and Experimental Medicine, University of Pisa, Italy.

Irene Mannari, The BioRobotics Institute, Scuola Superiore Sant'Anna, Pisa, Italy.

Martina Maselli, The BioRobotics Institute, Scuola Superiore Sant'Anna, Pisa, Italy.

Valentina Menici, IRCCS Fondazione Stella Maris, Calambrone, Pisa, Italy.

Filomena Paternoster, AOU "A. Meyer" Firenze, Italy.

We also thank Vincent Corsentino for the English review.

\section{Funding}

The Italian Ministry of Health project RF 2013-02358095 funded the present work.

\section{Authors' contributions}

GC is the principal investigator. GC, GS, MG and RR designed the clinical study. MG and RR are in charge for observational phase enrolment and infant data collection in Pisa (MG) and Florence (RR). GC and GS have the role of recruitment and randomization of infants in intervention phase. GS and $E B$ are responsible of $C T$ training and EB also of IM training. GC, GS and EB created the draft of the present manuscript. All authors have read and revised the manuscript and agreed on the final version.

\section{Authors' information}

GS is child neurologist and researcher at University of Pisa and at IRCCS Fondazione Stella Maris. EB is paediatric physical therapist at IRCCS Fondazione Stella Maris. MG is neonatologist involved in follow-up program at Neonatology Unit of Pisa University Hospital (AOUP). RR is child neuropsychiatrist involved in the follow-up program at University Children's Hospital "A. Meyer" in Florence. GC is full professor of child neuropsychiatry at University of Pisa and Scientific Director of IRCCS Fondazione Stella Maris.

\section{Ethics approval and consent to participate}

Tuscan Region Paediatric Ethics Committee (Italy) approved the present study (no. 84/2017). After receiving verbal and written informative material concerning the trial, eligible parents will be asked to fill out two informed consent forms at the start each of the two trial phases. The first one is relative to the observation phase while the second one to the intervention phase. The latter will be provided only to parents of non-fidgety infants. Both consent forms will be signed by parents or legal representative of eligible infants.

\section{Consent for publication}

Not applicable.

\section{Competing interests}

The authors declare that they have no competing interests.

\section{Publisher's Note}

Springer Nature remains neutral with regard to jurisdictional claims in published maps and institutional affiliations.

\section{Author details}

'Department of Developmental Neuroscience, IRCCS Fondazione Stella Maris, Viale del Tirreno 331, Calambrone, 56128 Pisa, Italy. ${ }^{2}$ Department of Clinical and Experimental Medicine, University of Pisa, Via Roma, 56125 Pisa, Italy. ${ }^{3}$ Neonatal Intensive Care Unit, Pisa University Hospital "Santa Chiara", Via Roma 67, 56126 Pisa, Italy. "Neuroscience Center of Excellence and Neonatal Intensive Care Unit, "A. Meyer" University Children's Hospital, Florence, Italy.

\section{Received: 30 April 2018 Accepted: 22 August 2018}

Published online: 05 September 2018

\section{References}

1. Behrman RE, Butler AS. Preterm Birth: Causes, Consequences, and Prevention. Washington (DC): National Academies Press (US); 2007. Committee on Understanding Premature Birth and Assuring Healthy Outcomes, Washington (DC): National Academies Press (US); 2007

2. Mclntyre S, Morgan C, Walker K, Novak I. Cerebral palsy-don't delay. Dev Dis Res Rev. 2011;17(2):114-29.
3. Cioni G, Sgandurra G, Muzzini S, Paolicelli PB, Ferrari A. Forms of Hemiplegia The Spastic Forms of Cerebral Palsy: A Guide to the Assessment of Adaptive Functions. Milano: Springer Milan; 2010. p. 331-56.

4. Novak I. Evidence-based diagnosis, health care, and rehabilitation for children with cerebral palsy. J Child Neurol. 2014;29(8):1141-56.

5. Bosanquet Copeland L, Ware R, Boyd R. A systematic review of tests to predict cerebral palsy in young children. Dev Med Child Neurol. 2013;55(5):418-26.

6. Novak I, Morgan C, Adde L, Blackman J, Boyd RN, Brunstrom-Hernandez J, Cioni G, Damiano D, Darrah J, Eliasson AC, de Vries LS, Einspieler C, Fahey M, Fehlings D, Ferriero DM, Fetters L, Fiori S, Forssberg H, Gordon AM, et al. Early, accurate diagnosis and early intervention in cerebral palsy: advances in diagnosis and treatment. JAMA Pediatr. 2017;171(9):897-907. https://doi. org/10.1001/jamapediatrics.2017.1689.

7. Cioni G, Sgandurra G. Normal psychomotor development in Handbook of Clinical Neurology on Pediatric Neurology. In: di Dulac O, Sarnat H, Lassonde M, editors. Amsterdam: Elsevier; 2013. vol. 111. p. 3-15.

8. Cioni $G$, Inguaggiato $E$, Sgandurra $G$. Early intervention in neurodevelopmental disorders: underlying neural mechanisms. Dev Med Child Neurol. 2016:58(Suppl 4):61-6.

9. Inguaggiato E, Sgandurra G, Cioni G. Brain plasticity and early development implications for early intervention in neurodevelopmental disorders. Neuropsychiatr Enfance Adolesc. 2017;65(5):299-306.

10. Battini R, Guzzetta A, Sgandurra G, Di Pietro R, Petacchi E, Mercuri E, Giannini MT, Leuzzi V, Cioni G. Scale for evaluation of movement disorders in the first three years of life. Pediatr Neurol. 2009;40(4):258-64.

11. Spittle A, Orton J, Anderson P, Boyd R, Doyle LW. Early developmental intervention programmes post-hospital discharge to prevent motor and cognitive impairments in preterm infants. Cochrane Database Syst Rev. 2012;12:CD005495

12. Morgan C, Darrah J, Gordon AM, Harbourne R, Spittle A, Johnson R, Fetters L. Effectiveness of motor interventions in infants with cerebral palsy: a systematic review. Dev Med Child Neurol. 2016;58(9):900-9. https://doi.org/ 10.1111/dmcn.13105. Epub 2016 Mar 29

13. Morgan C, Novak I, Badawi N. Enriched environments and motor outcomes in cerebral palsy: systematic review and meta-analysis. Pediatrics. 2013;132(3):e735-46.

14. Golomb McDonald BC, Warden SJ, Yonkman J, Saykin AJ, Shirley B, Huber M, Rabin B, Abdelbaky M, Nwosu ME, Barkat-Masih M, Burdea GC. In-home virtual reality videogame telerehabilitation in adolescents with hemiplegic cerebral palsy. Arch Phys Med Rehabil. 2010;91(1):1-8.

15. Sgandurra G, Bartalena L, Cioni G, Greisen G, Herskind A, Inguaggiato $E$, Lorentzen J, Nielsen JB, Sicola E, CareToy Consortium. Home-based, early intervention with mechatronic toys for preterm infants at risk of neurodevelopmental disorders (CARETOY): a RCT protocol. BMC Pediatr. 2014;14:268. https://doi.org/10.1186/1471-2431-14-268.

16. Cecchi F, Serio SM, Del Maestro M, Laschi C, Sgandurra G, Cioni G, Dario P. Design and development of "biomechatronic gym" for early detection of neurological disorders in infants. Conf Proc IEEE Eng Med Biol Soc. 2010; 2010:3414-7. https://doi.org/10.1109/IEMBS.2010.5627886.

17. Passetti G, Cecchi F, Baldoli I, Sgandurra G, Beani E, Cioni G, Laschi C, Dario $P$. Sensorized toys for measuring manipulation capabilities of infants at home. Conf Proc IEEE Eng Med Biol Soc. 2015;2015:7390-3. https:/doi.org/ 10.1109/EMBC.2015.7320099.

18. Rihar A, Sgandurra G, Beani E, Cecchi F, Pašič J, Cioni G, Dario P, Mihelj M. Munih M CareToy: stimulation and assessment of preterm Infant's activity using a novel Sensorized system. Ann Biomed Eng. 2016:44(12):3593-605. Epub 2016 Jun 10

19. Sgandurra G, Bartalena L, Cecchi F, Cioni G, Giampietri M, Greisen G, Herskind A Inguaggiato E, Lorentzen J, Nielsen JB, Orlando M. Dario P and CareToy consortium, a pilot study on early home based intervention through an intelligent baby gym (CareToy) in preterm infants. Res Dev Disabil. 2016;53-54:32-42.

20. Sgandurra $G$, Lorentzen J, Inguaggiato $E$, Bartalena L, Beani E, Cecchi $F$, Dario P, Giampietri M, Greisen G, Herskind A, Nielsen JB, Rossi G, Cioni G, CareToy Consortium. A randomized clinical trial in preterm infants on the effects of a home-based early intervention with the CareToy system. PLoS One. 2017;12(3):e0173521.

21. Bennett C, Underdown A, Barlow J. Massage for promoting mental and physical health in typically developing infants under the age of six months. Cochrane Database Syst Rev. 2013:4:CD005038.

22. Vickers A, Ohlsson A, Lacy JB, Horsley A. Massage for promoting growth and development of preterm and/or low birth-weight infants (review). Cochrane Database Syst Rev. 2004;2:CD000390. 
23. Abdallah B, Badr LK, Hawwari M. The efficacy of massage on short and long term outcomes in preterm infants. Infant Behav Dev. 2013;36(4):662-9.

24. Hernandez Reif M, Field T, Diego M, Beutler J. Evidence-based medicine and massage. Pediatrics. 2001 Oct;108(4):1053.

25. Einspieler C, Prechtl HF, Bos A, Ferrari F, Cioni G. Prechtl's method on the qualitative assessment of general movements in preterm, term and young infants (clinics in developmental medicine). London: MacKeith Press; 2005.

26. Einspieler C, Marschik PB, Bos AF, Ferrari F, Cioni G, Prechtk HFR. Early markers for cerebral palsy: insights from the assessment of general movements. Future Neurol. 2012;7(6):709-17.

27. Pagnozzi AM, Dowson N, Doecke J, Fiori S, Bradley AP, Boyd RN, Rose S. Identifying relevant biomarkers of brain injury from structural MRI: Validation using automated approaches in children with unilateral cerebral palsy. PLoS One. 2017;12(8):e0181605. https://doi.org/10.1371/journal.pone.0181605. eCollection 2017

28. George JM, Fiori S, Fripp J, Pannek K, Guzzetta A, David M, Ware RS, Rose SE, Colditz PB, Boyd RN. Relationship between very early brain structure and neuromotor, neurological and neurobehavioral function in infants born $<31$ weeks gestational age early hum dev. Early Hum Dev. 2018;117:74-82.

29. Fiori S, Guzzetta A, Pannek K, Ware RS, Rossi G, Klingels K, Feys H, Coulthard A, Cioni G, Rose S. Boyd RN validity of semi-quantitative scale for brain MRI in unilateral cerebral palsy due to periventricular white matter lesions: relationship with hand sensorimotor function and structural connectivity. Neuroimage Clin. 2015;8:104-9. https://doi.org/10.1016/.nicl.2015.04.005. eCollection 2015

30. Squires J, Bricker D (Eds): Ages \& stages questionnaires ${ }^{\ominus}$, 3rd edition. (ASQ$3^{\mathrm{TM}}$ ). A parent-completed child-monitoring system. Baltimore, MD: Paul Brookes; 2009

31. Heineman KR, Middelburg KJ, Bos AF, Eidhof L, La Bastide-Van Gemert S, Van Den Heuvel ER, Hadders-Algra M. Reliability and concurrent validity of the infant motor profile. Dev Med Child Neurol. 2013;55(6):539-45.

32. Provost B, Heimerl S, McClain C, Kim NH, Lopez BR, Kodituwakku P. Concurrent validity of the Bayley scales of infant development II motor scale and the Peabody developmental motor Scales-2 in children with developmental delays. Pediatr Phys Ther. 2004;16(3):149-56.

33. Bayley N, editor. Bayley scales of infant and toddler development ${ }^{\oplus}$ technical manual. 3rd ed. San Antonio, Texas: Pearson; 2005.

34. Teller DY, McDonald MA, Preston K, Sebris SL, Dobson V. Assessment of visual acuity in infants and children: the acuity card procedure. Dev Med Child Neurol. 1986;28(6):779-89.

35. Teller D, editor. Teller Acuity Card Manual. Dayton, Ohio, USA: Vistech Consultants Inc; 1990.

36. Guzzetta A, Baldini S, Bancale A, Baroncelli L, Ciucci F, Ghirri P, Putignano E, Sale A, Viegi A, Berardi N, Boldrini A, Cioni G, Maffei L. Massage accelerates brain development and the maturation of visual function. J Neurosci. 2009; 29(18):6042-51.

37. Pratesi A, Cecchi F, Beani E, Sgandurra G, Cioni G, Laschi C, Dario P. A new system for quantitative evaluation of infant gaze capabilities in a wide visual field. Biomed Eng Online. 2015;14:83. https://doi.org/10.1186/s12938-015-0076-7.

38. Crittenden PM, Claussen AH. Adaptation to varied environments. In: Crittenden PM, Claussen AH, editors. The organization of attachment relationships: maturation, culture, and context. New York: Cambridge University Press; 2002. p. 234-50.

39. Biringen Z. The universal language of love: assessing relationships through the science of emotional availability. United States of America: EA Press; 2009.

40. So K, Adamson TM, Horne RSC. The use of actigraphy for assessment of the development of sleep/wake patterns in infants during the first 12 months of life. J Sleep Res. 2007;16(2):181-7.

41. Greenspan SI, editor. Greenspan social-emotional growth chart: a screening questionnaire for infants and young children. San Antonio, Texas: Harcourt Assessment; 2004.

42. Abidin RR, editor. Parenting stress index. Odessa, Florida: Psychological Assessment Resources; 1995.

43. Sgandurra G, Cecchi F, Serio SM, Del Maestro M, Laschi C, Dario P, Cioni G. Longitudinal study of Unimanual actions and grasping forces during infancy. Infant Behav Dev. 2012;35(2):205-14.

44. Weller C, McNeil J. CONSORT 2010 statement: updated guidelines can improve wound care. J Wound Care. 2010;19:347-53.

45. Schulz KF, Altman DG. Moher D, for the CONSORT group: CONSORT 2010 statement: updated guidelines for reporting parallel group randomised trials. Ann Int Med. 2010;152(11):726-32.

\section{Ready to submit your research? Choose BMC and benefit from:}

- fast, convenient online submission

- thorough peer review by experienced researchers in your field

- rapid publication on acceptance

- support for research data, including large and complex data types

- gold Open Access which fosters wider collaboration and increased citations

- maximum visibility for your research: over $100 \mathrm{M}$ website views per year

At $\mathrm{BMC}$, research is always in progress.

Learn more biomedcentral.com/submissions 